\title{
INTERPRETATION OF LARGE-SCALE DEVIATIONS FROM THE HUBBLE FLOW ${ }^{1}$ \\ B. Grinstein, ${ }^{2}$ H. David Politzer, S.-J. Rey, ${ }^{3}$ and Mark B. Wise ${ }^{4}$ \\ California Institute of Technology \\ Received 1986 June 20; accepted 1986 September 9
}

\begin{abstract}
The theoretical expectation for large-scale streaming velocities relative to the Hubble flow is expressed in terms of statistical correlation functions. Only for objects that trace the mass would these velocities have a simple cosmological interpretation. If some biasing effects the objects' formation, then nonlinear gravitational evolution is essential to predicting the expected large-scale velocities, which also depend on the nature of the biasing.

Subject headings: cosmology — galaxies: redshifts
\end{abstract}

\section{INTRODUCTION}

An independent determination of the distance and the radial velocity of a large number of objects distributed out to the distances of order $50 h^{-1} \mathrm{Mpc}$, combined with knowledge of our own motion relative to the cosmic $3 \mathrm{~K}$ background radiation, allows a statistical determination of some sort of average velocity relative to the $3 \mathrm{~K}$ radiation that is somehow representative of the whole region (Rubin et al. 1976; Aaronson et al. 1986; Burstein et al. 1986, Collins, Joseph, and Robertson 1986). Such a net velocity may be of cosmological interest if large-scale velocity fluctuations are due to the accelerations produced by large-scale density fluctuations, since it then provides a measure of those density fluctuations (Clutton-Brock and Peebles 1981; Kaiser 1983; Vittorio and Silk 1985; Vittorio, Juszkiewicz, and Davis 1986). The purpose of this paper is to clarify with what statistical, theoretical expectations the observed average velocities should be compared. We argue that only if the objects trace the mass (and also under some even less likely hypotheses) is the comparison free of the complexities of nonlinear gravitational evolution. Hence, the magnitude and the variance of the expected net velocity for objects that do not trace the mass cannot be easily deduced for a particular cosmological model. Furthermore, observations done on different types of objects can be expected to yield different net velocities.

The magnitude of an observed net velocity is often compared with a statistically expected mean square velocity given by

$$
\begin{aligned}
\left\langle[v(R)]^{2}\right\rangle & \equiv\left\langle\left[\int d \boldsymbol{r} v(\boldsymbol{r}) W_{R}(\boldsymbol{r})\right]^{2}\right\rangle \\
& =\int d \boldsymbol{r} W_{R}(\boldsymbol{r}) \int d \boldsymbol{r}^{\prime} W_{R}\left(\boldsymbol{r}^{\prime}\right)\left\langle\boldsymbol{v}(\boldsymbol{r}) \cdot v\left(\boldsymbol{r}^{\prime}\right)\right\rangle .
\end{aligned}
$$

The velocity field $v(r)$ describes all the matter, treated theoretically as a fluid. $W_{R}(\boldsymbol{r})$ is a normalized weighting function of effective radius $R$ that defines the observed volume; its edges

1 Work supported in part by US Department of Energy under contract DEAC03-81-ER40050.

2 Tolman Fellow.

${ }^{3}$ Korea Foundation for Advanced Studies Graduate Fellow.

${ }^{4}$ Alfred P. Sloan Foundation Fellow, supported in part by US Department of Energy Outstanding Junior Investigator Program under contract DEFG03-84 ER40172. should be fuzzy, so that its Fourier transform falls off rapidly with wavenumber when $R$ is large. For sufficiently large $R$, the Fourier transform of the $\boldsymbol{v}-\boldsymbol{v}$ correlation is well approximated by its value in first-order linear gravitational perturbation theory. All further corrections are suppressed by powers of $1 / R$ as $R \rightarrow \infty$. Thus, a measurement of equation (1) would give information about primordial large-scale fluctuations, uncomplicated by nonlinearities in the time evolution.

Such dominance of primordial fluctuations over all nonlinear effects at large scales depends somewhat on the primordial mass density fluctuations. For example, it would not be true for fluctuations with a power spectrum that vanishes like $k^{4}$ as $k \rightarrow 0$. Throughout this paper, when we do a particular calculation, we assume that the primordial fluctuations are Gaussian with a Zel'dovich power spectrum $k^{1}$ (Zel'dovich 1972; Harrison 1970; Peebles and $\mathrm{Yu}$ 1970) and that the universe is at critical density, although many of our conclusions follow under more general circumstances.

In the following, we argue that the observed net velocity of a large ball cannot in general be compared with quantities such as the expression in (1). The problem is that the observed points (galaxies or clusters) are not randomly dispersed flotsam and jetsam, moving with random velocities superposed on some large-scale average. Rather, the positions of the observed points are correlated with fluctuations in the underlying density field $\rho(\boldsymbol{r})$, which, in turn, are correlated with the velocity fluctations. The average of the observed velocities is related to integrals over other correlation functions in addition to the $\boldsymbol{v}-\boldsymbol{v}$ correlation. In general, these additional terms receive contributions of the same order in $R$ and of comparable magnitude from each order in gravitational perturbation theory. Only under special statistical hypotheses are the additional terms negligible for large $R$ : this happens if the observed points trace the mass, if the observed positions are totally uncorrelated with the velocity field, or if the only connected correlation linking velocities and positions is a two-point function. If any nonlinear bias relates the distribution of the objects to the underlying density field, then gravitational evolution will ensure that the more general analysis is necessary, even for arbitrarily large $R$.

This paper is organized as follows. After reviewing the geometry of the observations, we identify the general statistical quantity that represents the expected mean square value for an observed net velocity. We discuss the conditions under which this reduces to equation (1) for large $R$. We give a simple 
example of how biased formation of the observed objects leads to essential contributions from nonlinear gravitational evolution. Hence we expect that different biasing criteria that lead to the formation of different types of luminous objects will receive different contributions from nonlinear gravity. Under the assumption that all classes of objects share a common velocity field (that of mass density), we show that the large-scale velocities of different classes are each proportional to the underlying $v(R)$. In the preceding examples we assume that the objects "go with the flow." i.e., their velocities are representative of the underlying fluid velocity at their location. We study the velocities of objects that form at peaks of the primordial density field as an example where this is not precisely the case. For point objects which would yield equation (1) for large $R$, we give an estimate of how large $R$ must be for the probability distribution of the velocity to be Gaussian by computing the connected four-point correlation of $v$ averaged over a Gaussian ball of large radius $R$.

\section{GEOMETRY OF OBSERVATIONS}

Using an independent distance determination to subtract out the Hubble flow component for each observed object from its observed radial velocity, one determines a radial velocity $\boldsymbol{v}_{\boldsymbol{i}} \cdot \hat{\boldsymbol{r}}_{i}$ for each object, labeled by $i$, relative to the Hubble flow, where $\hat{\boldsymbol{r}}_{i}=\boldsymbol{r}_{i} /\left|\boldsymbol{r}_{i}\right|$ and $\boldsymbol{r}_{i}$ is its position relative to us. The net velocity $v_{R}$ of the observed objects, $i=1, \ldots, N$, is defined as the result of the least-squares fit to the hypothesis that all object are moving with a single, common velocity $\boldsymbol{v}_{R}$. It follows that

$$
v_{R}^{\alpha}=\frac{3}{N} \sum_{i=1}^{N}\left(\boldsymbol{v}_{i} \cdot \hat{\boldsymbol{r}}_{i}\right) \hat{r}_{i}^{\beta} \cdot\left[\mathbf{M}^{-1}\right]^{\alpha \beta},
$$

where $\mathbf{M}^{\alpha \beta}$ is the $3 \times 3$ matrix

$$
\mathbf{M}^{\alpha \beta} \equiv \frac{3}{N} \sum_{i=1}^{N} \hat{r}_{i}^{\alpha} \hat{r}_{i}^{\beta}
$$

and repeated vector indices are summed in the usual fashion. If the survey is isotropic and $N$ is large, then, as the effective depth $R$ becomes large, $N$ can be expected to have only small fluctuations about its expected value $\bar{N}$, and $\mathbf{M}$ will be close to the identity matrix. Henceforth, we will ignore the fluctuations of $N$ and $\mathbf{M}$ about their large- $R$ values and take

$$
v_{R} \approx \frac{3}{\bar{N}} \sum_{i=1}^{N}\left(v_{i} \cdot \hat{r}_{i}\right) \hat{r}_{i}
$$

(Keeping $N$ and $\mathbf{M}$ explicitly in the subsequent discussions would lead to additional terms suppressed by powers of $1 / R$ as $R \rightarrow \infty$.)

To relate equation (4) for the observed net velocity $v_{R}$ to continuum concepts, it is convenient to introduce the field

$$
n(\boldsymbol{r}) \equiv \sum_{i} \delta^{3}\left(\boldsymbol{r}-\boldsymbol{r}_{i}\right)
$$

which describes the positions of all such objects in all of space, and imagine a field $v(r)$ such that

$$
v\left(r_{i}\right)=v_{i}
$$

Then, one can rewrite equation (4) as

$$
v_{R}^{\alpha} \equiv \frac{1}{\bar{n}} \int d \boldsymbol{r n}(\boldsymbol{r}) v^{\beta}(\boldsymbol{r}) \cdot \boldsymbol{W}_{\boldsymbol{R}}^{\alpha \beta}(\boldsymbol{r}),
$$

where $\bar{n}$ is the mean density of objects, and

$$
\boldsymbol{W}_{R}^{\alpha \beta}(\boldsymbol{r}) \equiv 3 W_{R}(\boldsymbol{r}) \hat{r}^{\alpha} \hat{r}^{\beta}
$$

here $W_{R}(r)$ is the normalized weight function that describes the observed region (just as was introduced in eq. [1]). Hence

$$
\left\langle\boldsymbol{v}_{R}^{2}\right\rangle=\frac{1}{\bar{n}^{2}} \int d \boldsymbol{r} \boldsymbol{W}_{R}^{\alpha \beta}(\boldsymbol{r}) \int d \boldsymbol{r}^{\prime} \boldsymbol{W}_{R}^{\alpha \gamma}\left(\boldsymbol{r}^{\prime}\right)\left\langle n(\boldsymbol{r}) v^{\beta}(\boldsymbol{r}) n\left(\boldsymbol{r}^{\prime}\right) v^{\gamma}\left(\boldsymbol{r}^{\prime}\right)\right\rangle .
$$

In Appendix A we show that the matrix structure of $W_{R}(r)$ implies a $1 / k^{6}$ tail in Fourier space for the weighting of $\langle(n v)(\boldsymbol{k})(n v)(-\boldsymbol{k})\rangle$, no matter how sharply $W_{R}(\boldsymbol{r})$ itself falls off in Fourier space.

\section{WHEN ARE CORRELATIONS OF $n(\boldsymbol{r}) \boldsymbol{v}(\boldsymbol{r})$ EQUIVALENT TO THOSE OF $\boldsymbol{v}(\boldsymbol{r})$ ?}

Under what conditions does the large- $R$ behavior of $\left\langle\boldsymbol{v}_{R}^{2}\right\rangle$ in equation (9) reduce to simply a $v-v$ correlation as in equation (1)? If $n(\boldsymbol{r})$ is totally without correlations, then the $n(\boldsymbol{r})$ 's cancel exactly with the $\bar{n}$ 's in the denominator in any expectation. If $n(\boldsymbol{r})$ is correlated but there exist no cross-correlations relating $n$ to $v$, then $\langle n v n v\rangle$ factors into $\langle n n\rangle\langle v v\rangle$, and for large separations $\left\langle n(\boldsymbol{r}) n\left(\boldsymbol{r}^{\prime}\right)\right\rangle / \bar{n}^{2}$ goes to unity. In both these cases the large- $R$ behavior of equation (9) trivially goes over to a $v$-v correlation.

Let the fluctuations of $n(\boldsymbol{r})$ be denoted by $\delta(\boldsymbol{r})$, i.e.,

$$
n(\boldsymbol{r})=\bar{n}[1+\delta(\boldsymbol{r})] \text {. }
$$

Then

$$
\begin{aligned}
\frac{1}{\bar{n}^{2}}\left\langle n(\boldsymbol{r}) \boldsymbol{v}(\boldsymbol{r}) n\left(\boldsymbol{r}^{\prime}\right) \boldsymbol{v}\left(\boldsymbol{r}^{\prime}\right)\right\rangle & =\left\langle\boldsymbol{v}(\boldsymbol{r}) \boldsymbol{v}\left(\boldsymbol{r}^{\prime}\right)\right\rangle+\left\langle\delta(\boldsymbol{r}) \boldsymbol{v}(\boldsymbol{r}) \boldsymbol{v}\left(\boldsymbol{r}^{\prime}\right)\right\rangle \\
& +\left\langle\boldsymbol{v}(\boldsymbol{r}) \delta\left(\boldsymbol{r}^{\prime}\right) \boldsymbol{v}\left(\boldsymbol{r}^{\prime}\right)\right\rangle+\left\langle\delta(\boldsymbol{r}) \boldsymbol{v}(\boldsymbol{r}) \delta\left(\boldsymbol{r}^{\prime}\right) \boldsymbol{v}\left(\boldsymbol{r}^{\prime}\right)\right\rangle .
\end{aligned}
$$

If the only nonzero connected correlation that relates $n$ and $v$ is $\left\langle\delta(\boldsymbol{r}) \boldsymbol{v}\left(\boldsymbol{r}^{\prime}\right)\right\rangle$ for $\boldsymbol{r} \neq \boldsymbol{r}^{\prime}$, then the $\langle\delta \boldsymbol{v} v\rangle$ terms in equation (11) vanish because $\langle\delta\rangle=\langle\boldsymbol{v}\rangle=0$; and the $\langle\delta \boldsymbol{v}\rangle\langle\delta \boldsymbol{v}\rangle$ term will give a vanishing contribution relative to the $\langle\boldsymbol{v} v\rangle$ term in the limit of large $R$.

To say that the observed points trace the mass means that the large-scale statistics of their distribution field $n(\boldsymbol{r})$ are linearly related to the statistics of the underlying mass density $\rho(\boldsymbol{r})$ and that the statistics of their velocity field $v(r)$ are a good approximation to those of the underlying fluid. Specifically, the connected correlations of the $n$ fluctuations $\delta(\boldsymbol{r})$ defined by equation (10) are the same as the connected correlations of the mass density fluctuation $\epsilon(\boldsymbol{r})$ defined by

$$
\rho(\boldsymbol{r})=\bar{\rho}[1+\epsilon(\boldsymbol{r})] ;
$$

and, in addition, the $v$ correlations for the two cases are the same. The action of gravity (beyond linear perturbation theory) induces all the relevant types of connected correlations, so that none of the particular hypotheses mentioned earlier in this section apply. Nevertheless, for objects that trace the mass, the $\langle\boldsymbol{v} v\rangle$ term dominates equation (11) for large $R$ to all orders in gravitational perturbation theory; the argument goes as follows.

The low-k behavior of $\tilde{\rho} \widetilde{v}(\boldsymbol{k})$ is curl-free (i.e., $\boldsymbol{k} \times \tilde{\rho} \widetilde{v}(\boldsymbol{k})=0$ ) to all orders in gravitational perturbation theory. In the notation of Goroff et al. (1986) this occurs because at low $\boldsymbol{k}$ the two-point function for $\widetilde{\rho}(\boldsymbol{k})$ is dominated by diagrams of the type shown in Figure 1. In Figure 1 the large crosshatched circles represent a subdiagram with an arbitrary number of 

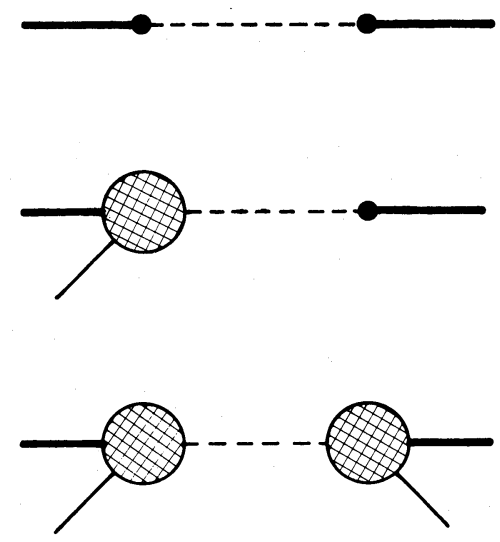

Fig. 1.-Diagrams which, according to the power counting arguments presented in Goroff et al. (1986), dominate the low- $k$ behavior of the two-point correlation of $\tilde{\rho} \widetilde{v}(\boldsymbol{k})$.

dotted lines. The dotted line connecting the two hatched circles carries the small wave vector $\boldsymbol{k}$. Rotational invariance implies that the integration over the wave vectors associated with the dotted lines in the hatched circle gives $\left\langle\widetilde{\rho} v^{i}(\boldsymbol{k}) \rho \widetilde{v}^{j}(-\boldsymbol{k})\right\rangle \propto k^{i} k^{j}$. Since $\widetilde{\rho} \boldsymbol{v}(\boldsymbol{k})$ is curl-free, at small $\boldsymbol{k}, \widetilde{\rho} \widetilde{v}(\boldsymbol{k})$ can be determined from $\tilde{\rho}$ using the expanding universe analog of the equation of continuity

$$
\tilde{\rho \boldsymbol{v}}(\boldsymbol{k})=\frac{i \boldsymbol{k}}{k^{2}} \frac{\partial \tilde{\rho}}{\partial t}(\boldsymbol{k}) .
$$

The two-point correlation of $\tilde{\rho}(\boldsymbol{k})$, at small $\boldsymbol{k}$, is dominated by the primordial fluctuation spectrum, independent of nonlinear gravitational evolution. The two point correlation of $\tilde{\boldsymbol{v}}(\boldsymbol{k})$ is identical (apart from the normalization factor $\bar{\rho}$ ) with the $\tilde{\rho} \widetilde{\boldsymbol{v}}(\boldsymbol{k})$ two-point correlation in linear perturbation theory, and at small $\boldsymbol{k}$ it is also unaltered by higher order gravitational effects. Hence, for the mass distribution itself (or for points that trace the mass), a measurement of $\rho(\boldsymbol{r}) \boldsymbol{v}(\boldsymbol{r})$ over a large region is as good as a measurement of $v(r)$ itself. (We have checked explicitly that for large $R$ the $\langle\epsilon \boldsymbol{v v}\rangle$ and $\langle\epsilon \boldsymbol{v} \in \boldsymbol{v}\rangle$ terms vanish relative to the $\langle\boldsymbol{v} v\rangle$ terms in the analog of eq. [11] to the first nontrivial order in gravitational perturbation theory.)

For objects that trace the mass, equation (9) can be compared with observations if the distance $R$ is so large that the probability distribution of $\boldsymbol{v}_{R}$ is approximately Gaussian. In the remainder of this section we examine this issue by computing the first two moments of the probability distribution for

$$
\tilde{\boldsymbol{V}}(R) \equiv \frac{\int d \boldsymbol{r} e^{-\boldsymbol{r}^{2} / R^{2}} \boldsymbol{v}(\boldsymbol{r})}{\int d \boldsymbol{r} e^{-\boldsymbol{r}^{2} / R^{2}}}=\int \frac{d k}{(2 \pi)^{3}} e^{-k^{2} R^{2} / 4} \tilde{\boldsymbol{v}}(\boldsymbol{k}) .
$$

Connected correlations of $v(R)$ at large $R$ are determined by the tree graphs of gravitational perturbation theory. For the four-point function, the graphs which contribute are shown in Figure 2. A useful measure of whether the probability distribution is Gaussian is the quantity

$$
r(R)=\frac{\left\langle v(R)^{2} v(R)^{2}\right\rangle_{\text {connected }}}{(5 / 3)\left\langle v(R)^{2}\right\rangle^{2}\left\langle\epsilon(R)^{2}\right\rangle},
$$

where $\epsilon(R)$ is the mass density fluctuation averaged over the Gaussian ball of radius $R$. The quantity $r(R)$ is independent of the normalization of the primordial fluctuations and approaches a nonzero constant as $R \rightarrow \infty$. We have computed $r(R)$ for cold dark matter, and the results are shown in Figure 3.
For any reasonable normalization of the primordial fluctuations [i.e., with $\left\langle\epsilon(R)^{2}\right\rangle<5$ at $R h^{2}=5 \mathrm{Mpc}$ ] the probability distribution appears to be Gaussian [i.e., the connected fourpoint correlation $\left\langle\boldsymbol{v}(R)^{2} v(R)^{2}\right\rangle_{\text {connected }}$ is significantly less than the disconnected piece $\left.(5 / 3)\left\langle\boldsymbol{v}(R)^{2}\right\rangle\left\langle\boldsymbol{v}(R)^{2}\right\rangle\right]$ for $R h^{2}>5 \mathrm{Mpc}$.

\section{BIASED FORMATION}

Since the objects recently surveyed -clusters, ellipticals and spirals - each as a class appear to have qualitatively different spatial distributions, it is highly unlikely that they all statistically trace the total mass distribution. It is even quite possible that none of them do, and the criteria for the formation of the individual objects in each class involve some different nonlinear biasing relative to the primordial density fluctuations. If so, then $\delta(r)$, the fluctuation in their number distribution defined in equation (10), is some nonlinear functional of $\epsilon(\boldsymbol{r})$, the total mass density fluctuation defined in equation (12). Under such circumstances, general power counting arguments for gravitational perturbation theory imply that each term in equation (11) has the same leading dependence on $R$, and, furthermore, the terms with both $\delta$ and $v$ receive contributions of comparable magnitude from each order in perturbation theory.

In the special case of points that trace the mass, the equation of continuity played a crucial role in ensuring that each of these purported leading additional corrections to the primordial $\langle\boldsymbol{v} v\rangle$ in fact has a zero coefficient. There is no reason for such a cancellation for a general $\delta(\boldsymbol{r})$, and we have checked that the simplest imaginable nonlinear biasing gives this effect. In particular, we consider the case of

$$
\delta(\boldsymbol{r})=\epsilon(\boldsymbol{r})+\alpha \epsilon(\boldsymbol{r})^{2}-a\left\langle\epsilon(\boldsymbol{r})^{2}\right\rangle,
$$

which can be thought of as a term in an expansion for weak biasing of some more general functional. In Appendix B we show explicitly that the relevant terms do not cancel in the lowest nontrivial order of gravitational perturbation theory.

It is because various fields are evaluated at the same point that each term in equation (11) scales with the same power of $R$ as $R \rightarrow \infty$. If the position of each field were integrated over the observed volume independently, then the $\langle\boldsymbol{v} v\rangle$ term would
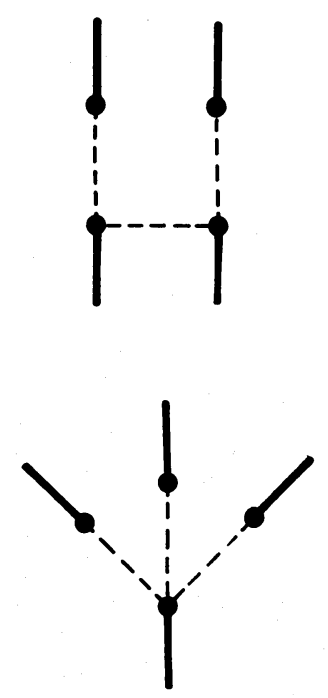

Fig. 2.-Tree graphs which give the dominant contribution to the connected four-point correlation of $v(R)$.

\section{(C) American Astronomical Society - Provided by the NASA Astrophysics Data System}




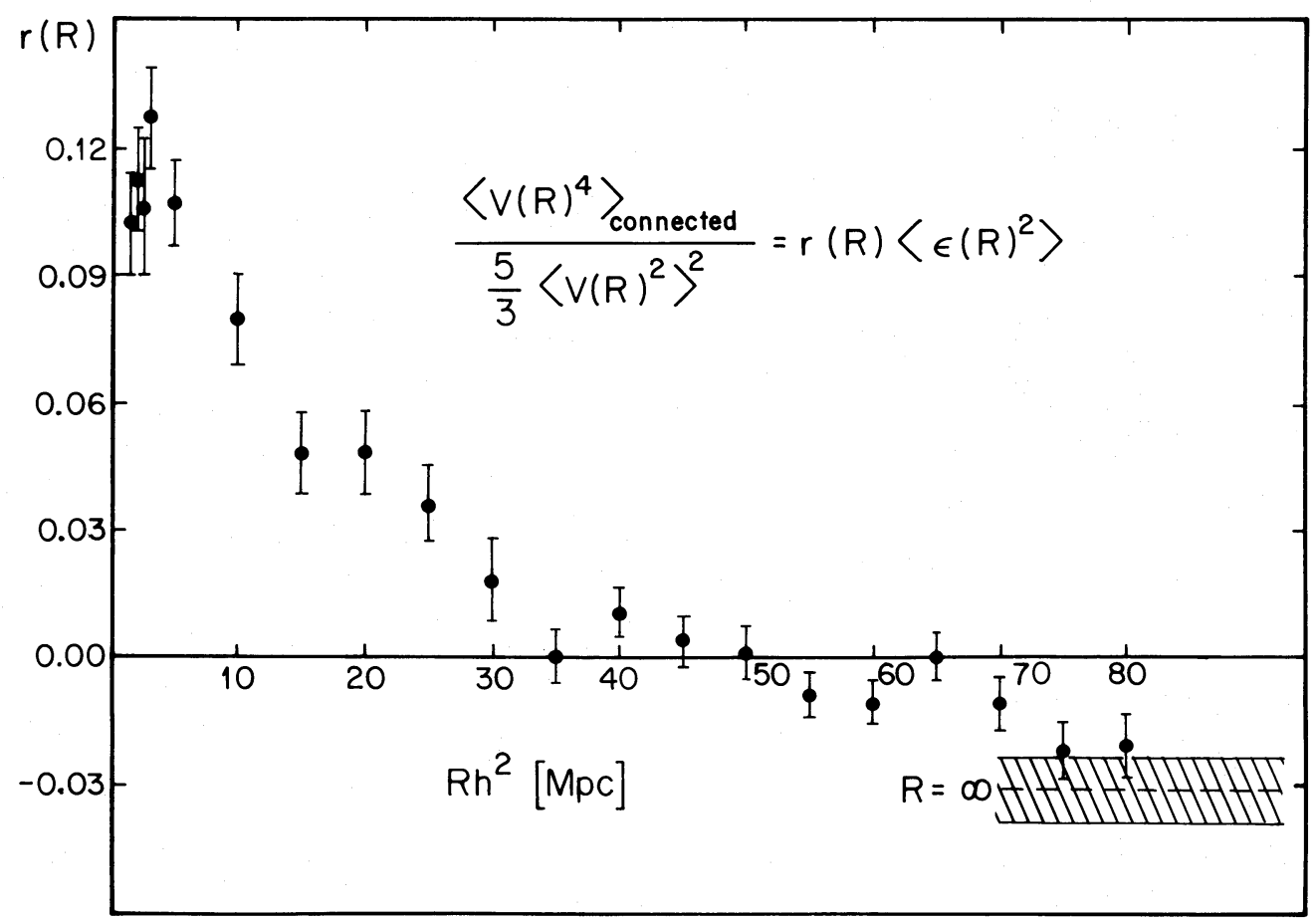

Fig. 3.-Plot of $r(R)$ versus $R h^{2}$ in Mpc. The error bars are $1 \sigma$ errors incurred in the Monte Carlo evaluation of integrals. The shaded $(1 \sigma)$ region gives the value of $r(R)$ as $R \rightarrow \infty$.

indeed dominate. Also, for the same reason, each order in perturbation theory makes a comparable contribution.

The crucial issue can be described in another fashion. Gravitational perturbation theory is an expansion in powers of $\epsilon_{1}(r)$, the primordial density fluctation described as a linear perturbation. It is small at each point only at very early times, but its average over sufficiently large volumes remains small for very long times. When "point" objects are observed, the "point" corresponds to a volume in space which is the region which has collapsed to form the object. On that scale, $\epsilon(\boldsymbol{r})$ may have grown sufficiently large to go nonlinear. Therefore, $\epsilon(r)^{2} v(r)$ need not be small relative to $v(r)$ at the location of the collapsed object. [Note, however, that in a model where objects form only at very high peaks the typical $\epsilon(r)$ has not necessarily gone nonlinear on the scale of collapsing objects, and linear perturbation theory may still be valid for $n v$.]

From a graphical analysis, we conclude that, for large $R, v_{R}$ becomes a Gaussian random variable (i.e., the connected parts of its correlations fall off faster than the disconnected parts as $R \rightarrow \infty)$, but the magnitude of $\left\langle\boldsymbol{v}_{R}^{2}\right\rangle$ cannot be deduced from linear perturbation theory. Only its $R$ dependence can be inferred from power counting all orders of perturbation theory. The integrals over just the $\langle\boldsymbol{v} v\rangle$ term may give the correct order of magnitude, but they cannot be used for a quantitative comparison of observation with cosmological theory.

Could a volume-averaged velocity such as appears in equation (1) be deduced somehow from observations? The answer would be yes only if the point objects approximated a fluid, i.e., if their mean separation were small compared to the distance over which $\delta(\boldsymbol{r})$ and $\boldsymbol{v}(\boldsymbol{r})$ correlations change appreciably. Were that the case, then the observed volume could be divided into cells such that the correlations would be approximately constant over each cell while the cells would be large enough to ensure that each would contain at least a few objects. Only under these conditions would one find

$$
\frac{\int_{\text {cell }} d r n(r) v(r)}{\int_{\text {cell }} d r n(r)} \approx \frac{\int_{\text {cell }} d r v(r)}{\int_{\text {cell }} d r} \equiv v_{\text {cell }} .
$$

However, all classes of luminous objects have too low a density relative to the variation of their correlation functions to allow this procedure for measuring $v(r)$ independent of $\delta(r) v(r)$.

We learn about the relative orientations of the net velocities of different classes of objects produced by different biasing criteria by studying the expectation of their dot product:

$$
\begin{aligned}
\left\langle v_{1}(R) \cdot v_{2}(R)\right\rangle=\frac{1}{\bar{n}_{1} \bar{n}_{2}} \int & d r_{1} W_{R}\left(r_{1}\right) \int d r_{2} W_{R}\left(r_{2}\right) \\
& \times\left\langle n_{1}\left(r_{1}\right) v\left(r_{1}\right) \cdot n_{2}\left(r_{2}\right) v\left(r_{2}\right)\right\rangle .
\end{aligned}
$$

The object densities $n_{1}$ and $n_{2}$ could be $1, \rho(\boldsymbol{r})$ or any nonlinear (local) function of the primordial mass density fluctuations $\epsilon(\boldsymbol{r})$, but we assume that the objects labeled 1 and 2 trace the underlying velocity field $\boldsymbol{v}(\boldsymbol{r})$. Under the assumption of Gaussian primordial statistics with a Zel'dovich spectrum, the analysis of Goroff et al. (1986) allows us to conclude that the leading diagrams of gravitational perturbation theory as $R \rightarrow \infty$ are all of the structure indicated in Figure 4. The heavy lines represent the $v$ 's, and thin lines represent the appropriate factor of $\epsilon$ in $n_{1}$ and $n_{2}$; the dotted line represents the primordial linearized

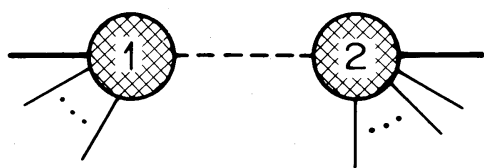

Fig. 4.- The diagram structure that dominates the leading $R \rightarrow \infty$ behavior of $\int d r_{1} W_{R}\left(r_{1}\right) \int d r_{2} W_{R}\left(r_{2}\right)\left\langle n_{1}\left(r_{1}\right) v\left(r_{1}\right) \cdot n_{2}\left(r_{2}\right) v\left(r_{2}\right)\right\rangle$. 
fluctuation. In terms of wave vectors, the dotted line carries a small $\boldsymbol{k}$ which is integrated with $\left|\widetilde{W}_{R}\right|^{2}$, while the other lines are integrated over all wave vectors that add up to $\boldsymbol{k}$. The crosshatched circles include arbitrarily complex vertices.

The form of Figure 4 implies that equation (18) has a factorized structure as $R \rightarrow \infty$, i.e.,

$$
\left\langle\boldsymbol{v}_{1}(R) \cdot \boldsymbol{v}_{2}(R)\right\rangle=B(R) c_{1} c_{2}+\cdots,
$$

where $c_{1}$ and $c_{2}$ are the values of the crosshatched circles as $k \rightarrow 0$ and $B(R)$ is independent of the particular choices of $n_{1}$ and $n_{2}$. Equation (19) implies that

$$
\left\langle\left[c_{2} v_{1}(R)-c_{1} v_{2}(R)\right]^{2}\right\rangle=0
$$

to leading order in $1 / R$. Since equation (20) is an average of squares, the cancellation must hold for each observation even without averaging over an ensemble, i.e.,

$$
c_{2} v_{1}(R)=c_{1} v_{2}(R) \text {. }
$$

The nonleading contributions to equation (19) do not have the same factorization property and lead to fluctuations of $c_{2} v_{1}(R)$ relative to $c_{1} v_{2}(R)$, but these vanish relative to $v_{1}(R)$ and $v_{2}(R)$ as $R \rightarrow \infty$.

The simplest choice for $n_{2}$ is simply 1 . So we learn that all large-scale velocities, given our various assumptions, are proportional to the primordial large-scale velocity of the mass, whose value is determined by linear perturbation theory. It is the constant of proportionality that gets essential contributions from all scales and all orders of perturbation theory.

The conclusion that different classes of objects (all of which go with the flow) can yield net velocities of different magnitudes, even for arbitrarily large regions, is contrary to the common interpretation that such large-scale average velocities represent the collective infall toward some supermassive fluctuation. In such a picture, any large sample of objects should yield the common, average infall velocity produced by the common large-scale component of the acceleration. This represents a possible configuration, but our analysis suggests that, statistically, small-scale fluctuations play an important role. The expectation for the magnitude of an observed net $v_{R}$ is related to the statistical question of how well the individual point velocities cancel for $R \neq \infty$, and the degree of the noncancellation depends both on fluctuations on all scales and on the selection criteria that define the points.

\section{THE VELOCITY OF LOCAL MAXIMA OF THE MASS DENSITY FIELD}

We have assumed in the previous discussions that the velocity field of the observed objects matches that of the total matter distribution. For objects that trace the mass this is true by definition, but it is not obviously a necessary property for all objects. As an example, we consider objects that form at local maxima of the mass density field (Peacock and Heavens 1985; Bardeen et al. 1986) and identify the objects' velocities with the velocities of the local maxima. We show below that in gravitational perturbation theory those velocities are not identical with the velocities of the matter field evaluated at the local maxima. Which of these two velocities is, in fact, a better indicator of the final velocity of the hypothesized collapsed object is at present a matter of conjecture. The answer requires a better understanding of galaxy formation than is currently available.

In gravitational perturbation theory the fluctuations in the mass density field $\epsilon(\boldsymbol{r}, t)$ and the peculiar velocity field $\boldsymbol{v}(\boldsymbol{r}, t)$ have expansions

$$
\begin{aligned}
& \epsilon(r, t)=\epsilon_{1}(r)\left(t / t_{0}\right)^{2 / 3}+\epsilon_{2}(r)\left(t / t_{0}\right)^{4 / 3}+\cdots, \\
& v(r, t)=\frac{a(t)}{t}\left(\frac{t}{t_{0}}\right)^{2 / 3} v_{1}(r)+\frac{a(t)}{t}\left(\frac{t}{t_{0}}\right)^{4 / 3} v_{2}(r)+\cdots,
\end{aligned}
$$

where $\boldsymbol{r}$ is the comoving coordinate, $t_{0}$ is the present time, and $a(t)$ is the Robertson-Walker scale factor. Local maxima of the mass density field are located at comoving coordinates $\boldsymbol{r}_{\boldsymbol{m}}(t)$ determined by

$$
\nabla \epsilon\left(\boldsymbol{r}_{m}, t\right)=0,
$$

and the peculiar velocity of the local maxima is

$$
\boldsymbol{v}_{m}=a(t) \frac{d}{d t} \boldsymbol{r}_{m}(t)
$$

Note that $v_{m}$ is the peculiar velocity of the position $r_{m}$ and is in principle distinct from the peculiar velocity field evaluated at $\boldsymbol{r}_{m}$, i.e., $\boldsymbol{v}\left(\boldsymbol{r}_{m}, t\right)$. In linear perturbation theory only the first term in equations (22) are kept. Evidently, if only linear perturbation theory for the mass density fluctuations were used (i.e., the first term in eq. [22a]), the local maxima would occur at a fixed comoving coordinate $r_{m}^{(0)}$ determined by $\nabla \epsilon_{1}\left(r_{m}^{(0)}\right)=0$, and they would have no peculiar velocity. The comoving coordinate of a local maximum has an expansion

$$
r_{m}(t)=r_{m}^{(0)}+r_{m}^{(1)}\left(t / t_{0}\right)^{2 / 3}+\cdots
$$

We show below how the second term in equation (25) gives rise to a peculiar velocity for local maxima of the same order in $t / t_{0}$ as the peculiar velocity of the mass in linear perturbation theory.

To determine $\boldsymbol{r}_{m}^{(1)}$, we expand equation (23) using equations (22a) and (25), giving

$$
r_{m}^{(1)}=-\left[\mathbf{w}^{-1}\right]^{\alpha \beta} \nabla^{\beta} \epsilon_{2}\left(r_{m}^{(0)}\right),
$$

where the matrix $\mathbf{w}$ has components

$$
[\mathbf{w}]^{\alpha \beta}=\nabla^{\alpha} \nabla^{\beta} \epsilon_{1}\left(r_{m}^{(0)}\right) \text {. }
$$

From the explicit expression for $\epsilon_{2}$ (Peebles 1980) we deduce that

$$
\boldsymbol{r}_{m}^{(1)}=\frac{3}{2} \boldsymbol{v}_{1}-\frac{9}{14}\left[\mathbf{w}^{-1}\right] \nabla\left[\left(\nabla^{\alpha} v_{1}^{\beta}\right)\left(\nabla^{\alpha} v_{1}^{\beta}\right)\right]
$$

Equation (24) implies that the first term in equation (28) contributes to the local maximum velocity $\boldsymbol{v}_{m}$ an amount equal to the velocity of the mass density evaluated at the location of the local maximum. The second term in equation (28) arises from the fact that the peaks change shape with time, and this shifts the precise location of the local maximum. We have not explored whether the second term in equation (28) significantly affects correlations of $n_{m} v_{m}$ averaged over large scales.

\section{CONCLUSION}

When point objects are used to survey the flow within a region, it is really the volume average of $n(\boldsymbol{r}) \boldsymbol{v}(\boldsymbol{r})$ that is measured rather then the average of $v(r)$ directly, where $n(\boldsymbol{r})$ gives the locations of the objects whose velocity field is $v(r)$. For objects that trace the total mass distribution, the large volume average of $n(\boldsymbol{r}) \boldsymbol{v}(\boldsymbol{r})$ and of $v(\boldsymbol{r})$ would be equivalent, and their measurement would have a straightforward interpretation in 
terms of linearized gravitational evolution of primordial cosmological mass density fluctuations. However, for objects that do not trace the mass but instead correspond to some biased formation criteria and, hence, to some nonlinear relation to the total mass density, fluctuations induced by smallscale, nonlinear gravitational evolution are relevant. As the size of the observed region increases, the rate at which these fluctuations even out is no greater than the rate at which the fluctuations in $v(r)$ itself even out. Hence, the two cannot be separated, even for arbitrarily large regions. This phenomenon then precludes any simple comparison of the magnitudes of the observed net velocities of large regions with cosmological expectations. How such net velocities scale with the size of the observed region is predictable, given the primordial spectrum, but the overall coefficient, which could very well vary between different types of objects, is beyond present analytic computational capabilities. One prediction which can be compared directly with observation is whether the net velocities deduced from different classes of objects in a given volume are parallel. This prediction rests on the assumption that all objects trace the underlying flow, but given that hypothesis, the degree of alignment is a measure of whether the observed volume is really large enough for asymptotic $R \rightarrow \infty$ estimates to apply.

Given a particular model for the biasing, the behavior of the volume average of $n \boldsymbol{v}$ for different primordial mass density fluctuations could be deduced from computer simulations of the nonlinear evolution. However, one must note the following issue. The different velocities as represented by the different $c$ 's in equation (21) receive contributions from fluctuations on short scales. For a Zel'dovich primordial spectrum, there is a characteristic scale at which the spectrum ceases to go as $k^{1}$ and turns over. Physics at this scale will be important in determining the velocities of objects. Furthermore, to reproduce the effects we have discussed, the simulation would have to be sufficiently fine-grained to see the gravitational evolution to go nonlinear on the scale of the collapsed objects.

\section{APPENDIX A}

Here we exhibit the structure in Fourier transform space that follows from observing only the radial components of velocities. Recall that

$$
\begin{aligned}
v_{R}^{\alpha} & =\frac{1}{\bar{n}} \int d \boldsymbol{r} \boldsymbol{W}_{R}^{\alpha \beta}(\boldsymbol{r})\left(n v^{\beta}\right)(\boldsymbol{r}) \\
& =\frac{1}{\bar{n}} \int \frac{d \boldsymbol{k}}{(2 \pi)^{3}}\left(\widetilde{n v^{\beta}}\right)(-\boldsymbol{k}) \tilde{W}_{R}^{\alpha \beta}(\boldsymbol{k}) .
\end{aligned}
$$

Letting

$$
\tilde{W}_{R}^{\alpha \beta}(\boldsymbol{k})=3 \int d \boldsymbol{r} e^{i \boldsymbol{k} \cdot \boldsymbol{r}} \frac{r^{a} r^{\beta}}{r^{2}} W_{R}(\boldsymbol{r})=\delta^{\alpha \beta} A(\boldsymbol{k})+\frac{k^{a} k^{\beta}}{k^{2}} B(\boldsymbol{k})
$$

we see that

$$
A+\frac{1}{3} B=\int d r e^{i \boldsymbol{k} \cdot r} W_{R}(\boldsymbol{r})=\tilde{W}_{R}(\boldsymbol{k})
$$

and

$$
\frac{1}{3}(A+B)=\frac{1}{k^{2}} \int d r e^{i k \cdot r} \frac{(\boldsymbol{k} \cdot \boldsymbol{r})^{2}}{r^{2}} W_{R}(\boldsymbol{r}) .
$$

Performing the integration in equation (A4), we find that

$$
\frac{1}{3}(A+B)=-\frac{1}{6} \tilde{W}_{R}(\boldsymbol{k})+\frac{1}{4 \pi} \int \frac{d \boldsymbol{q}}{q^{3}}\left[1-3\left(\frac{\boldsymbol{k} \cdot \boldsymbol{q}}{k q}\right)^{2}\right] \tilde{W}_{R}(\boldsymbol{k}-\boldsymbol{q}) .
$$

Note that even if $\tilde{W}_{R}(q)$ is very sharply peaked about $q=0$, the second term in equation (A5) implies a $1 / k^{6}$ tail for the weighting of $\langle\widetilde{n}(\boldsymbol{k}) \widetilde{n} \widetilde{v}(-\boldsymbol{k})\rangle$ in the expectation $\left\langle\boldsymbol{v}_{R}^{2}\right\rangle$.

\section{APPENDIX B}

Here we compute the small- $k$ behavior of

$$
\left\langle\widetilde{\epsilon^{2} v^{i}}(\boldsymbol{k}) \tilde{v}^{j}\left(\boldsymbol{k}^{\prime}\right)\right\rangle_{\text {connected }}=f^{i j}(\boldsymbol{k})(2 \pi)^{3} \delta^{3}\left(\boldsymbol{k}+\boldsymbol{k}^{\prime}\right)
$$

to the lowest nontrivial order in gravitational perturbation theory. The graphs which give a contribution to $f^{i j}(k)$ that diverges

\section{(C) American Astronomical Society - Provided by the NASA Astrophysics Data System}



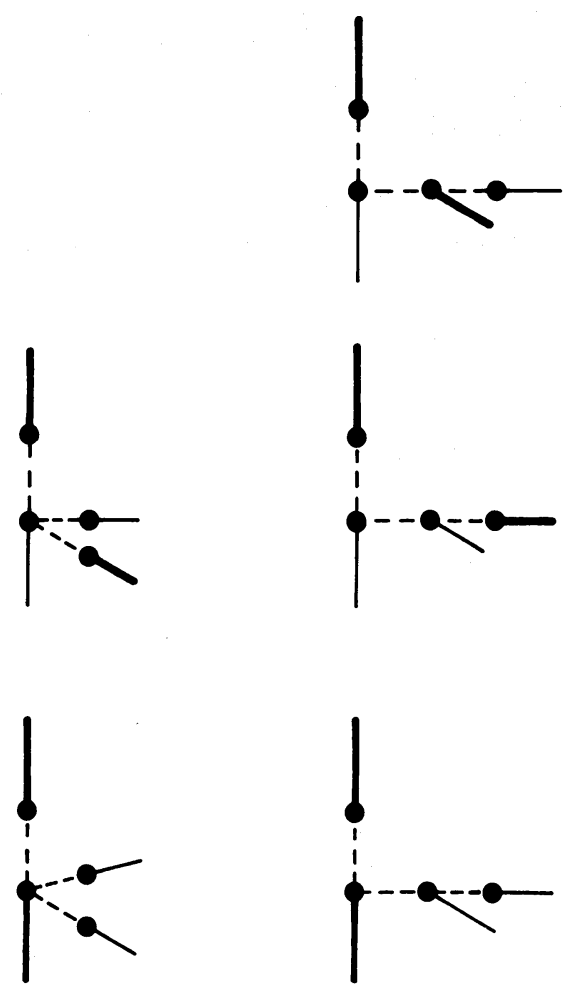

FIG. 5.-Diagrams which dominate the low- $k$ behavior of $f^{i j}(k)$ (see eq. [B1]) in the lowest nontrivial order of gravitational perturbation theory.

linearly as $\boldsymbol{k} \rightarrow 0$ are shown in Figure 5. They give

$$
\begin{aligned}
f^{i j}(\boldsymbol{k})= & \frac{4 k^{i} k^{j}}{9 k^{3}} \int \frac{d \boldsymbol{p}}{(2 \pi)^{3}} \int \frac{d \boldsymbol{q}}{(2 \pi)^{3}}\left\{-\frac{4}{3} A(p) A(|\boldsymbol{p}+\boldsymbol{q}|) p|\boldsymbol{p}+\boldsymbol{q}| \cdot P_{2}^{(s)}(-\boldsymbol{p}, \boldsymbol{p}+\boldsymbol{q})\right. \\
& +2 \frac{(\boldsymbol{p}+\boldsymbol{q}) \cdot \boldsymbol{q}}{q^{2}} p|\boldsymbol{p}+\boldsymbol{q}| A(p) A(|\boldsymbol{p}+\boldsymbol{q}|) \cdot Q_{2}^{(s)}(-\boldsymbol{p}, \boldsymbol{p}+\boldsymbol{q}) \\
& +\frac{4}{3} \frac{(\boldsymbol{p}+\boldsymbol{q}) \cdot \boldsymbol{p}}{p^{2}} p|\boldsymbol{p}+\boldsymbol{q}| A(p) A(|\boldsymbol{p}+\boldsymbol{q}|) P_{2}^{(s)}(-\boldsymbol{p}, \boldsymbol{p}+\boldsymbol{q}) \\
& \left.+\frac{4}{3} \frac{\boldsymbol{p} \cdot(\boldsymbol{p}+\boldsymbol{q})}{p^{2}} p q A(p) A(q) P_{2}^{(s)}(\boldsymbol{p}, \boldsymbol{q})-p q A(p) A(q) Q_{2}^{(s)}(\boldsymbol{p}, \boldsymbol{q})\right\}
\end{aligned}
$$

where

$$
\begin{aligned}
& P_{2}^{(s)}(\boldsymbol{p}, \boldsymbol{q})=\frac{5}{7}+\frac{p \cdot q}{2 p q}\left(\frac{p}{q}+\frac{q}{p}\right)+\frac{2}{7}\left(\frac{p \cdot q}{p q}\right)^{2} \\
& Q_{2}^{(s)}(p, q)=\frac{2}{7}+\frac{p \cdot q}{3 p q}\left(\frac{p}{q}+\frac{q}{p}\right)+\frac{8}{21}\left(\frac{p \cdot q}{p q}\right)^{2}
\end{aligned}
$$

and the primordial mass density fluctuations have a power spectrum

$$
\left\langle\tilde{\boldsymbol{\epsilon}}^{1}(\boldsymbol{k}) \tilde{\epsilon}_{1}\left(\boldsymbol{k}^{\prime}\right)\right\rangle=k A(k)(2 \pi)^{3} \delta^{3}\left(\boldsymbol{k}+\boldsymbol{k}^{\prime}\right) .
$$

With cold dark matter $A(k)$ falls as $(\ln k)^{2} / k^{4}$ for large $k$.

\section{REFERENCES}

Aaronson M., Bothun, G., Mould, J., Huchra, J., Schommer, R. A., and Cornell, M. E. 1986, Ap. J., 302, 536.

Bardeen, J. M., Bond, J. R., Kaiser, N., and Szalay, A. S. 1986, Ap. J., 304, 15.

Burstein, D., Davies, R. L., Dressler, A. Faber, S. M., Lynden-Bell, D., Terlevich, R., and Wegner, G. 1986, in Proc. NATO/ASI Meeting on Extra galactic Distance Scale and Deviations from Hubble Flow (Kona, Hawai).
Clutton-Brock, M., and Peebles, P. J. E. 1981, Astr. J., 86, 1115.

Collins, C. A., Joseph, R. D., and Robertson, N. A. 1986, Nature, $320,506$. Goroff, M. H., Grinstein, B., Rey, S. J., and Wise, M. B. 1986, Ap. J., 311, 6 Harrison, E. 1970, Phys. Rev. D., 1, 2726

Harrison, E. 1970, Phys. Rev. D., 1, 2726.
Kaiser, N. 1983, Ap. J. (Letters), 273, L17.

Peacock, J., and Heavens, A. 1985, M.N.R.A.S., 217, 805 
Peebles, P. J. E. 1980, The Large-Scale Structure of the Universe (Princeton: Princeton University Press)

Peebles, P. J. E., and Yu, J. 1970, Ap. J., 162, 815

, Rubin, V. C., Ford, W. K., Jr., Thonnard, N., Roberts, M. S., and Graham, J. A

ion 1976, A.J., 81, 687 .

$\lim _{1 \rightarrow 1}$

B. Grinstein, H. David Politzer, S.-J. Rey, and Mark B. Wise: California Institute of Technology, Pasadena, CA 91125

Vittorio, N., Juszkiewicz, R., and Davis, M. 1986, University of California, Berkeley, preprint.

Vittorio, N., and Silk, J. 1985, Ap. J. (Letters), 293, L1.

Zel'dovich, Y. 1972, M.N.R.A.S., 160, 18 